\title{
Sistema de vacío con bolsa de orina, variación técnica
}

\section{Vacuum System with Urine Bag, Technical Variation}

\author{
Walter Chávez Gárate ${ }^{1} \quad$ Eva Camba Gonzalez $^{1} \quad$ Jose Couceiro Otero $^{1}$ \\ 1 Unidad de Cirugía de la Mano, departamento de Cirugía Ortopédica y \\ Traumatología, Hospital POVISA, Vigo, España \\ Address for correspondence Walter Chávez Gárate, Calle Tarragona \\ 12 1-A - Vigo, Spain (e-mail: walter_chg@hotmail.com).
}

Rev Iberam Cir Mano 2017;45:45-49.

\section{Resumen \\ Palabras Clave \\ - terapia de vacío \\ - cicatrización de heridas \\ - técnicas de cierre de heridas}

\section{Abstract \\ Keywords \\ - negative-pressure wound therapy \\ - wound healing \\ - wound closure techniques}

En el presente trabajo se propone un método simple, económico y eficiente para la aplicación de la terapia de presión negativa en el tratamiento de heridas en cirugía de la mano. El procedimiento consiste en abrir el extremo distal de una bolsa colectora de orina, cortar su válvula interna, realizar la cobertura de la herida con esponjas, colocar un drenaje a través del tubo de la bolsa, sellar la bolsa y aplicar la presión negativa habitual. Esa variación para la colocación de la terapia de vacío, permite el tratamiento de heridas grandes y complejas en la mano, sobre todo aquellas que involucran los dedos, en las que obtener un sellado que no pierda el vacío, con los plásticos habituales, es complejo. Como ventaja adicional, se puede controlar la vascularización local al ser una bolsa transparente. También es posible realizar movilización y/o rehabilitación tempranas incluso con la terapia funcionando. Las posibles complicaciones son principalmente el realizar vacío sobre piel sana y la rara pero posible reacción local alérgica. Esa es una modificación técnica muy útil y fácil de realizar para obtener un vacío adecuado en una localización compleja.

In this paper a simple, inexpensive, and efficient method for the application of Negative Pressure Wound Therapy in hand surgery is presented. The procedure consists in opening the distal end of the urine bag, cutting the inner valve, covering the wound with the foam, placing a drainage through the tube of the bag, sealing the bag and applying the negative pressure through the tube of the bag like a traditional construct. This variation for placing the negative pressure wound therapy allows the treatment for large and complex hand wounds, especially those involving the digits in which obtaining the sealing that dont lose the vacuum, with the commonly used plastics, is complex. An additional advantage is that the local vascularization can be monitorized as the bag is transparent. It also allows an early mobilization and rehabilitation even with the therapy working. The possible complications are mainly the performance of the pressure on a healthy skin and rare but possible local allergic reactions. This is a useful technical modification, easy to perform to obtain an adequate vacuum in a complex location. received

October 26, 2016

accepted

April 4, 2017
DOI https://doi.org/

10.1055/s-0037-1602807. ISSN 1698-8396.
Copyright $\odot 2017$ by Thieme Revinter

Publicações Ltda, Rio de Janeiro, Brazil
License terms

(요 (1) $\Theta \circledast$ 


\section{Introducción}

El efecto beneficioso del uso de la presión negativa para el tratamiento de heridas en cirugía de la mano (Vacuum Asisted Closure: VAC) ha sido ampliamente descrito y corroborado en la literatura. ${ }^{1,2}$ Sabemos que la presión negativa aplicada sobre heridas, supone un estrés mecánico tisular que estimula la angiogénesis, disminuye la colonización bacteriana y aumenta la formación de tejido de granulación en las mismas. $^{2}$

Cuando aplicamos un sistema VAC en heridas de la mano, nos enfrentamos muchas veces a heridas complejas, con exposición ósea y/o tendinosa, así como lesiones asociadas que requieren una estrecha monitorización y delicada manipulación durante su recuperación. Además, una vez aplicada, y dada la anatomía de la mano, es inevitable dejar los dedos inmovilizados por los vendajes oclusivos, por lo que una terapia rehabilitadora muchas veces no es posible durante el tratamiento.

El sistema tradicional consta de esponjas de poliuretano o polininilo, aplicadas sobre una herida abierta y cubiertas con un vendaje semioclusivo, sometiendo a la esponja a presión negativa vía succión. ${ }^{2,3}$ Sin embargo, existen diversas modificaciones de la técnica original, que incluyen el uso de tiras adhesivas, bolsas selladas comerciales ${ }^{1}$ e incluso guantes transparentes ${ }^{3-5}$ para la aplicación de ese tipo de terapia, todas ellas con buenos resultados publicados.

La ventaja principal de las bolsas prefabricadas comercializadas sobre la técnica original radica en su mayor facilidad para aplicarlas y en la posibilidad de realizar fisioterapia temprana al permitir la movilidad de forma precoz.

Nosotros proponemos un método simple, económico y eficiente; desarrollado por las enfermeras de la unidad de cirugía de la mano en nuestro hospital, el cual requiere el uso de una bolsa colectora de orina estéril para la aplicación de la cobertura sellada y la posterior succión.

\section{Indicaciones y contraindicaciones}

El uso de una bolsa para aplicar la técnica VAC en la mano es muchas veces necesaria, especialmente en heridas extensas y complejas, en las cuales una manipulación limitada intraoperatoria y una monitorización meticulosa post quirúrgica son claves para un buen manejo terapéutico.

Las indicaciones clásicas de la terapia mediante presión negativa se aplican indistintamente a nuestro método, que incluyen: heridas asociadas a fracturas abiertas, heridas con o sin defectos de cobertura por partes blandas, heridas con exposición tendinosa, ósea, articular o de material de osteosíntesis, heridas infectadas después del lavado y desbridamiento. ${ }^{1,2}$

Ese método no requiere la compra o esterilización de ningún material específico, puesto que todos los componentes necesarios se encuentran disponibles en casi la totalidad de hospitales.

Nuestro diseño evita el uso de diversas capas de adhesivos, reduciendo el riesgo de fugas de vacío que ese método conlleva, simplificando su aplicación y minimizando la manipulación excesiva de la mano afectada. La bolsa que utilizamos, al ser transparente, permite una monitorización estrecha de la mano, sobre todo de su estado vascular. Así mismo, nos permite realizar movilización y/o rehabilitación temprana del miembro afectado, con tan solo suspender temporalmente la presión negativa.

Nosotros creemos además, que realizar una cobertura de heridas circunferenciales ya sea en el pulgar u otros dedos, es una situación técnicamente compleja y delicada; en la que la aplicación del VAC mediante tiras adhesivas simples, conlleva a una mayor manipulación, inadecuada aplicación y riesgo de fracaso. Situación en la cual el uso del VAC mediante una bolsa se nos presenta como ideal.

Las contraindicaciones de la terapia asistida por presión negativa son exposición de vasos, nervios, anastomosis, y tejido necrótico con presencia de una escara. ${ }^{1-3}$

\section{Técnica quirúrgica}

El procedimiento se realiza de la siguiente forma: Utilizamos una bolsa colectora de orina (en nuestro caso: Teleflex Medical Europe Ltd, Co. Westmeath Ireland) (-Fig. 1) a la cual realizamos un orificio en el extremo distal del tamaño requerido para la introducción de la mano del paciente dentro de la bolsa; retiramos la válvula interna de la misma (-Fig. 2) dado que tiende a colapsarse.

Luego, la mano cubierta con las correspondientes esponjas, es colocada dentro de la bolsa acompañada de un pequeño tubo de drenaje de $20-30 \mathrm{~cm}$ (en nuestro caso un drenaje número 10, de Iberhospitex, Barcelona, España) el cual, con un extremo sobre las esponjas, pasará a través de la válvula seccionada y se dejará el otro extremo dentro del tubo de la bolsa colectora. (-Figs. 2 y 3 )

Sellamos el orificio alrededor de la mano con un film adhesivo (nosotros usamos Opsite ${ }^{\circledR}$ (Smith and Nephew, Londres, Reino Unido) para conseguir un ambiente hermético (-Fig. 4). Se realiza una prueba para detectar posibles fugas en el sistema una vez terminado, aplicando finalmente la presión negativa mantenida a través del tubo de la bolsa, tal y como en la técnica habitual del VAC (-Fig. 5).

\section{Postoperatorio}

Se recomienda realizar curas y cambiar el sistema cada 72 horas como promedio. Mantenemos una presión continua de $-125 \mathrm{~mm} \mathrm{Hg}$, mediante un sistema fijo a pared, aunque no descartamos la posibilidad de utilizar un sistema portátil acoplado a la bolsa, que ya ha sido probado por otros autores. ${ }^{6}$

La labor de recambio resulta notablemente cómoda y sencilla con nuestro diseño, tanto como suspender la presión negativa y retirar los componentes necesarios para dicho procedimiento.

Como mencionamos anteriormente, es posible llevar a cabo la movilización activa y/o la rehabilitación temprana y controlada del miembro afectado, al suspender temporalmente la terapia de vacío pero sin retirar la bolsa, de forma 


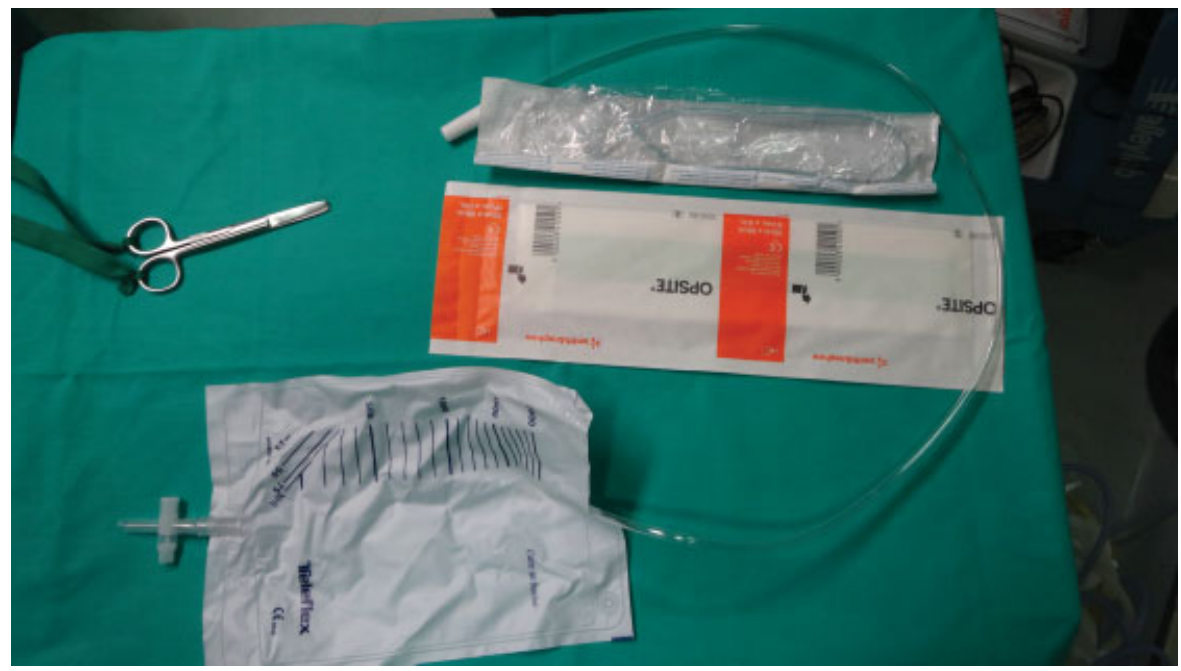

Fig. 1 Materiales: Bolsa de orina, tubo de drenaje y cubierta adhesiva.

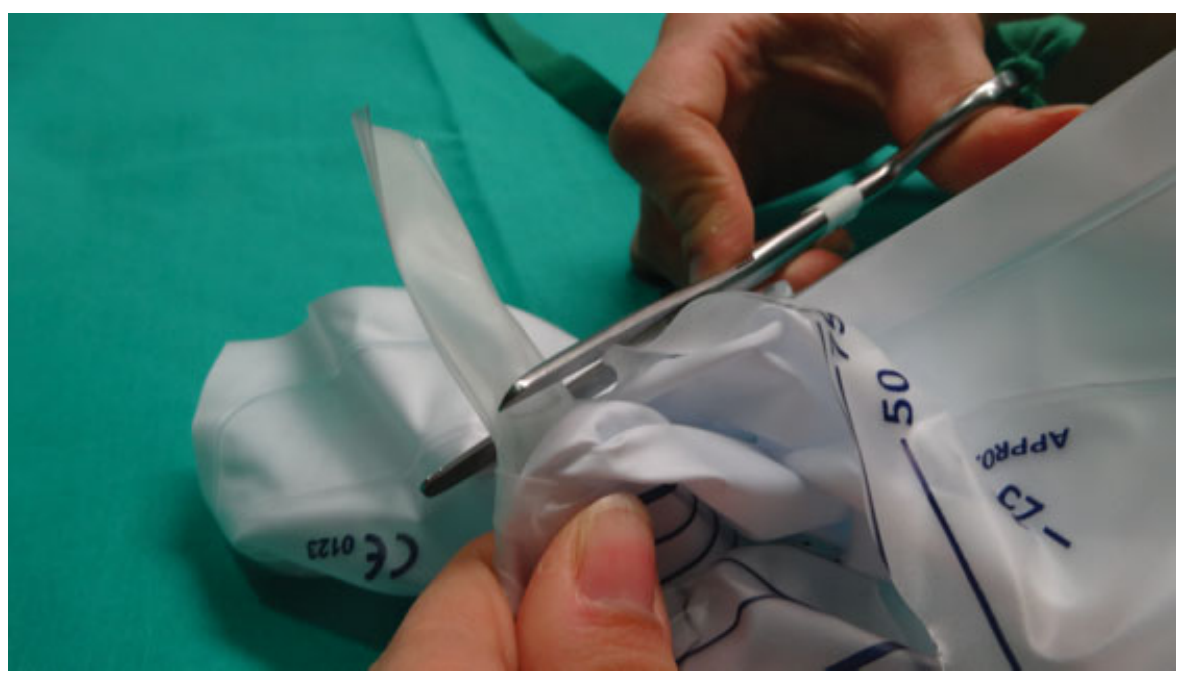

Fig. 2 La válvula interna es removida.

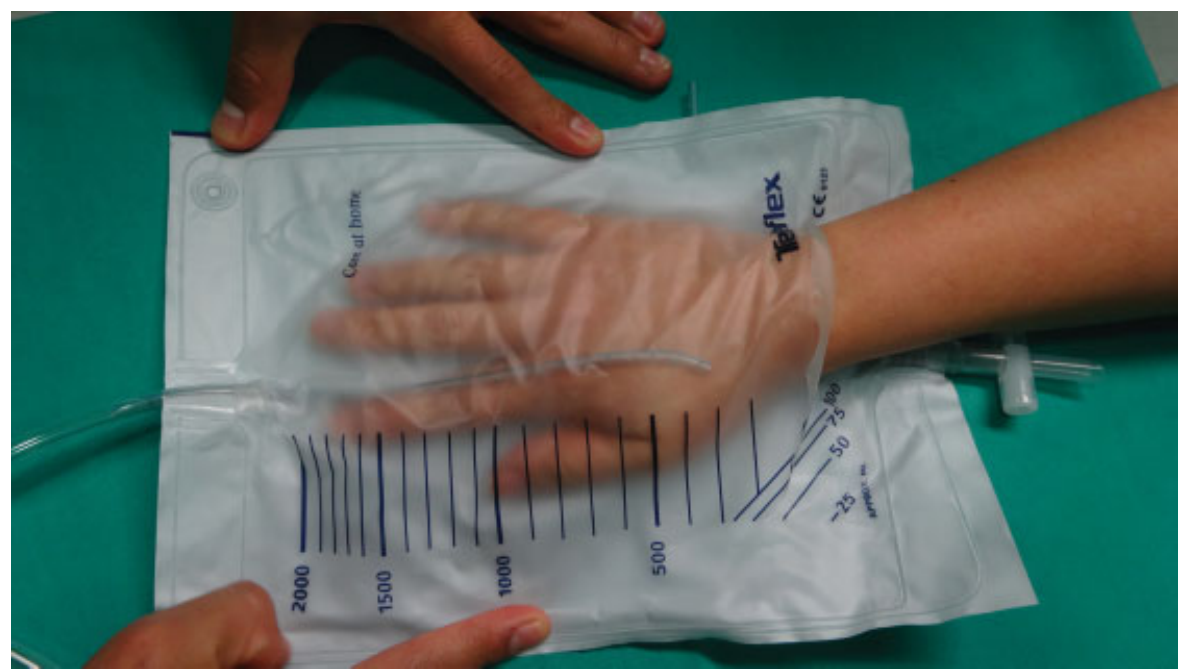

Fig. 3 La bolsa es abierta proximalmente para poder introducir la mano y las esponjas. El pequeño drenaje es colocado a través de la válvula por dentro de la bolsa, con un extremo sobre la esponja. 


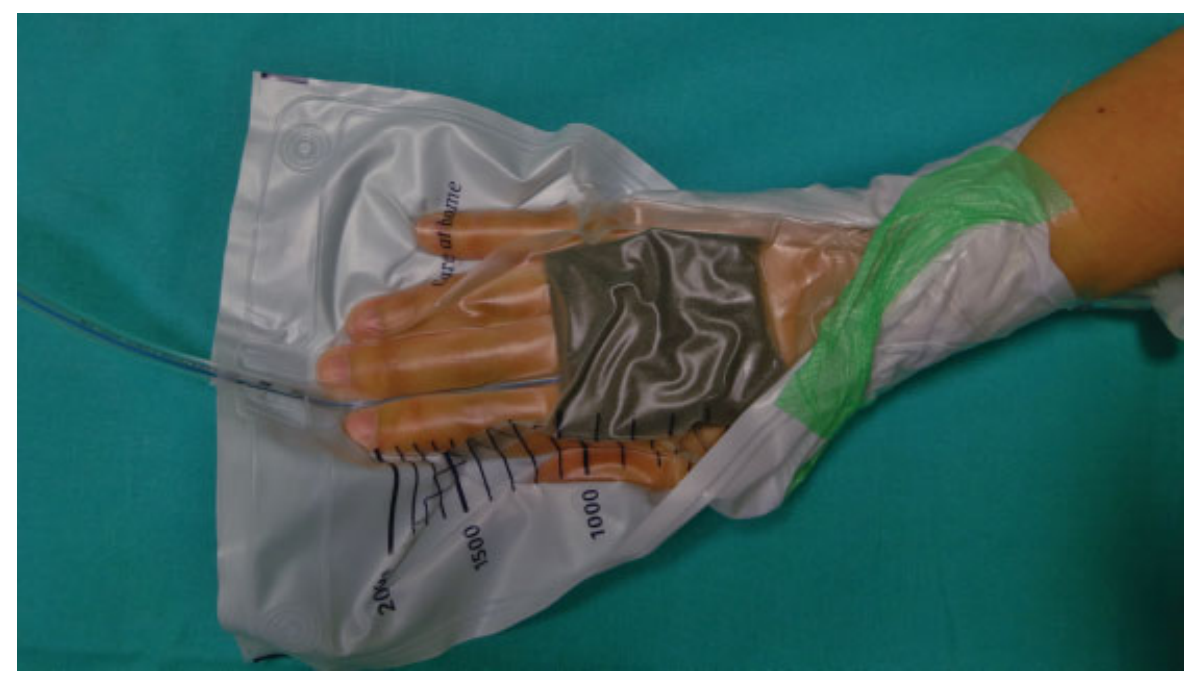

Fig. 4 Luego sellamos la bolsa con el film adhesivo y aplicamos presión negativa a través del drenaje de la bolsa de orina, probando el sistema ante eventuales fugas.

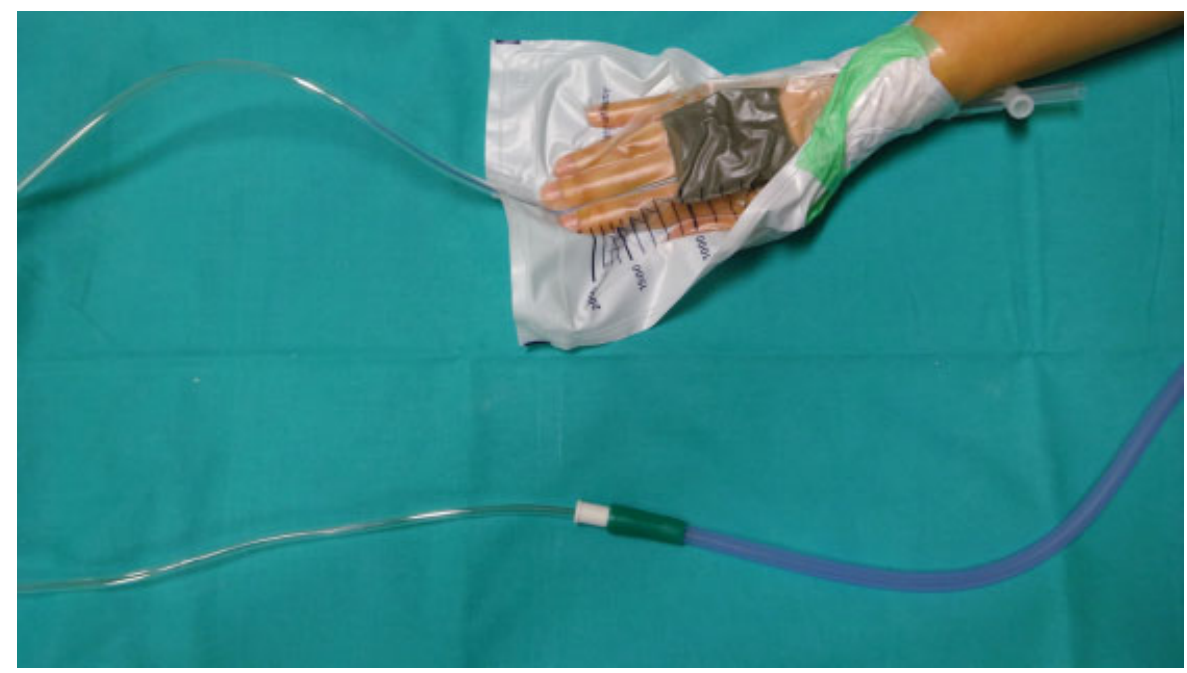

Fig. 5 Conectamos el tubo de la bolsa a la aspiración continua con la presión deseada.

que la presión negativa pueda ser reiniciada después de la terapia. La pauta rehabilitadora varía en función del tipo de traumatismo o herida presentada, siendo en el común de los casos, de inicio precoz (primeras 48-72h).

\section{Complicaciones}

Una complicación potencial de nuestro método es la cobertura secundaria, muchas veces inevitable, de zonas de piel sana, la cual puede macerarse debido a la humedad. El riesgo de maceración está presente en todas las modificaciones de la técnica VAC y puede reducirse al mínimo de distintas maneras: En primer lugar el cirujano debe asegurarse de la no existencia de fugas aéreas para mantener un ambiente adecuado, ya que la presión negativa aplicada en esas condiciones, parece permitir un drenaje adicional de cualquier exudado excesivo, por lo que en ese aspecto la bolsa podría ser ventajosa respecto al método tradicional. En segundo lugar, podemos proteger la piel sana mediante la aplicación de determinados apósitos o protectores (Por ejemplo DuoDerm $^{\circledR}$ ), los cuales son mucho más sencillos de emplear en una bolsa, que no se ve afectada por el volumen que significarían dichos apósitos, si la comparamos con los guantes o sistemas clásicos de VAC. En tercer lugar, debemos vigilar el estado cutáneo y detectar en forma temprana cualquier signo de maceración o compromiso de piel sana, lo cual se ve ampliamente facilitado por el diseño transparente de la bolsa.

Podemos considerar también la rara pero posible reacción local alérgica a los componentes de la bolsa (PVC no tóxico).

Las complicaciones clásicas descritas de la terapia VAC son lesiones cutáneas de sangrado e infección. Todas ellas relacionadas con la técnica de aplicación y recambio del sistema. $^{2}$ 


\section{Bibliografía}

1 Hasegawa K, Namba Y, Kimata Y. Negative pressure wound therapy incorporating early exercise therapy in hand surgery: bag-type negative pressure wound therapy. Acta Med Okayama 2013;67(04):271-276

2 Lesiak AC, Shafritz AB. Negative-pressure wound therapy. J Hand Surg Am 2013;38(09):1828-1832

3 Sommier B, Sawaya E, Pelissier P. The hand in the mitt: a technical tip for applying negative pressure in hand wounds. J Hand Surg Eur Vol 2014;39(08):896
4 Seyhan H, Kopp J, Polykandriotis E, Horch RE. [Vacuum-assisted closure as temporary coverage in the "problem zone hand"]. Zentralbl Chir 2006;131(Suppl 1):S33-S35

5 Fujitani T, Zenke Y, Shinone M, Menuki K, Fukumoto K, Sakai A. Negative Pressure Wound Therapy with Surgical Gloves to Repair Soft Tissue Defects in Hands. J UOEH 2015;37(03):185-190

6 Narayan N, Edwards D, Ragoowansi RR. Glove and PICO: A novel technique for treatment of chronic wound due to osteomyelitis of the hand. BMJ Case Rep 2014;2014; Doi: 10.1136/bcr-2013202780 